\title{
An investigation of the efficiency of pedicle screw simulator software in thoracic
}

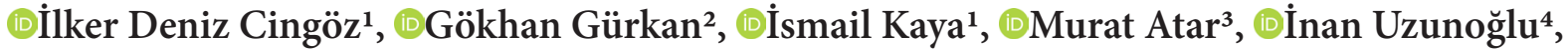 \\ (D) Meryem Cansu Şahin ${ }^{5}$, @Nurullah Yüceer ${ }^{2}$ \\ ${ }^{1}$ Usak University, Faculty of Medicine, Department of Neurosurgery, Ușak, Turkey \\ ${ }^{2}$ Katip Çelebi University, School of Medicine, Department of Neurosurgery, İzmir, Turkey \\ ${ }^{3}$ Sultan 2. Abdulhamid Han Training and Research Hospital, Department of Neurosurgery, İstanbul, Turkey \\ ${ }^{4}$ University of Health Sciences, Dıșkapı Yıldırım Beyazıt Training and Research Hospital, Department of Neurosurgery, Ankara, Turkey \\ ${ }^{5}$ Kutahya Health Sciences University, Research and Training Center, Kütahya, Turkey
}

Cite this article as: Cingöz İD, Gürkan G, Kaya İ, et al. An investigation of the efficiency of pedicle screw simulator software in thoracic. J Health Sci Med 2022; 5(1): 252-256.

\begin{abstract}
Aim: Fennell et al. describe a simple, effective freehand technique for thoracic pedicle screw insertion. In this study, we aimed to compare the patients undergoing upper thoracic pedicle screw insertion with the Fennell technique simulated on Pedicle Screw Simulator (PSS) and those recruited to the same procedure utilizing C-arm fluoroscopy.

Material and Method: First, we uploaded pre-operative CT images of 12 patients to the PSS module, which was used in our study to calculate the screw angle and visualize the pedicle screw entry point and trajectories. Then, we created three-dimensional vertebral models of the patients to simulate screw placement using visualization tool kit (VTK), open-source software for 3D computer graphics and visualization, available free of charge as part of 3D Slicer. Next, we placed pedicle screws through predetermined anatomic regions. C-arm fluoroscopy-guided pedicle screws were placed in the patients in the control group. The amount of bleeding, operation times and correct screw placement data were recorded in both groups.

Results: 24 patients were included in the study. The mean age of the patients was $32.3 \pm 4.1$ years. We applied 80 pedicle screws to Group 1 and 72 to Group 2. According to the malposition classification by Rao et al. on postoperative CTs, 68 patients in Group 1 were classified as Grade 0, 8 as Grade 1, 4 as Grade 2. Yet, there were no statistical differences between the groups by Rao et al.s classification $(\mathrm{p}>0.05)$. While the mean operation time of Group 1 was $138 \pm 34$ minutes, it was $162 \pm 44$ minutes in Group 2 . The groups significantly differed by operation time $(\mathrm{p}<0.05)$.

Conclusion: Overall, pre-operative simulation on PSS may allow more efficient and easier thoracic pedicle screw application. In addition, the simulator may contribute to the training of surgeons on upper thoracic pedicle screw application and increase the accuracy of pedicular screw placement.
\end{abstract}

Keywords: Freehand technique, pedicle screw simulator, upper thoracic pedicle screws

\section{INTRODUCTION}

Incorrect placement of pedicle screws can lead to serious complications (1-5). Pedicle screws can safely be inserted using intraoperative tools such as fluoroscopy and computed tomography-guided (CT) navigation (6). Yet, variations in vertebrae particularly make pedicle screw insertion difficult. Moreover, the use of navigation is not common in our country due to its high cost. In addition, surgeons have serious concerns about radiation exposure when using fluoroscopy.

Today, pre-operative simulation software serves to verify the reliability of various techniques in screw placement and is used in the training of spine surgeons (7-9). Accordingly, Pedicle Screw Simulator (PSS) is a versatile module that can be used for pre-surgical planning in 3D Slicer, an open-source platform developed for this purpose $(10,11)$.

Placement of an upper thoracic pedicle screw with fluoroscopy, but without a pre-operative navigation system, is also challenging for surgeons. It also requires a large number of fluoroscopy shots. To overcome this situation, Fennell et al. describe a simple, effective freehand technique of thoracic pedicle screw insertion using a uniform entry point for all levels (12). 
Ultimately, this study aimed to compare the patients undergoing upper thoracic pedicle screw insertion with the Fennell technique simulated on pedicle screw simulator (PSS) and those recruited to the same procedure utilizing $\mathrm{C}$-arm fluoroscopy.

\section{MATERIAL AND METHOD}

The study was carried out with the permission of Uşak University Non-Interventional Clinical Research Ethics Committee (Date: 23.09.2021, Decision No: 175-17509). All procedures were carried out in accordance with the ethical rules and the principles of the Declaration of Helsinki.

We recruited 24 patients for whom a surgical treatment (pedicle screw) was planned for a pathological fracture (non-traumatic) in the upper thoracic region (T1-T6 vertebrae). We applied pedicle screwing on 12 patients (Group 1) with the Fennell technique simulated on PSS, while the procedure was performed on 12 patients in the control group (Group 2) with the help of C-arm fluoroscopy.

Patients who underwent posterior transpedicular screw for upper thorax pathological fracture and gave informed consent were included in the study. Patients with a history of trauma and thoracic spine surgery were excluded from the study.

\section{Pre-operative Planning}

In our study, we utilized PSS (http://www.slicer.org, Surgical Planning Lab (SPL), Boston, USA) module on the 3D Slicer environment coded in Python language. We reconstructed CT data with a slice thickness of $1 \mathrm{~mm}$, a spacing of $1 \mathrm{~mm}$ between axial slices, and a matrix size of $512 \times 512$ for each slice.

We uploaded pre-operative CT images of Group 1 to the PSS module, which was used in our study to calculate the screw angle and visualize the pedicle screw entry point and trajectories. This method allowed the surgeon to finalize pre-operative planning in a short time. Then, we created three-dimensional vertebral models for the patients to simulate screw placement using VTK (Visualization Tool Kit), open-source software for 3D computer graphics and visualization, available free of charge as part of 3D Slicer (Figure 1).

Next, we set two trajectory planning modes using the respective vertebrae, with ET (Entry-Target mode) and EA (Entry-Angle mode) on the control panel. We then selected the desired vertebral level using the plus sign on the 3D Slicer toolbar (Figure 2).

In ET mode, we selected the entry and target reference points for the pedicle screw through axial, sagittal, and coronal CT series and the $3 \mathrm{D}$ vertebra model. Then, we calculated the pitch and deviation angles for the desired pedicle screw using the software and selected desired diameter of the pedicle screw on the PSS control panel. Afterward, we visualized the trajectory of the pedicle screw using the "place screw for given entry and target" button. The length of the screw was calculated on the program based on the Euclidean distance between the desired entry and target reference points (Figure 3).

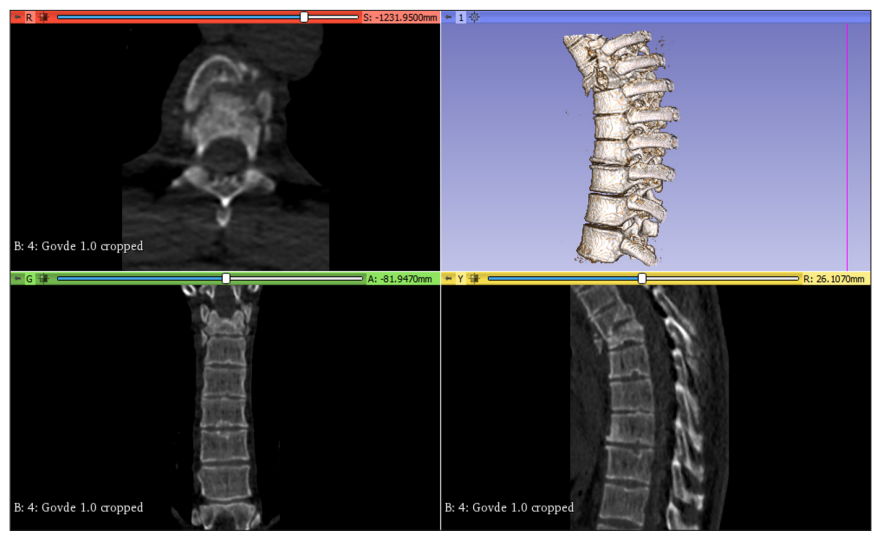

Figure 1.3D vertebra model on preoperative CT images extracted using Visualization Tool Kit

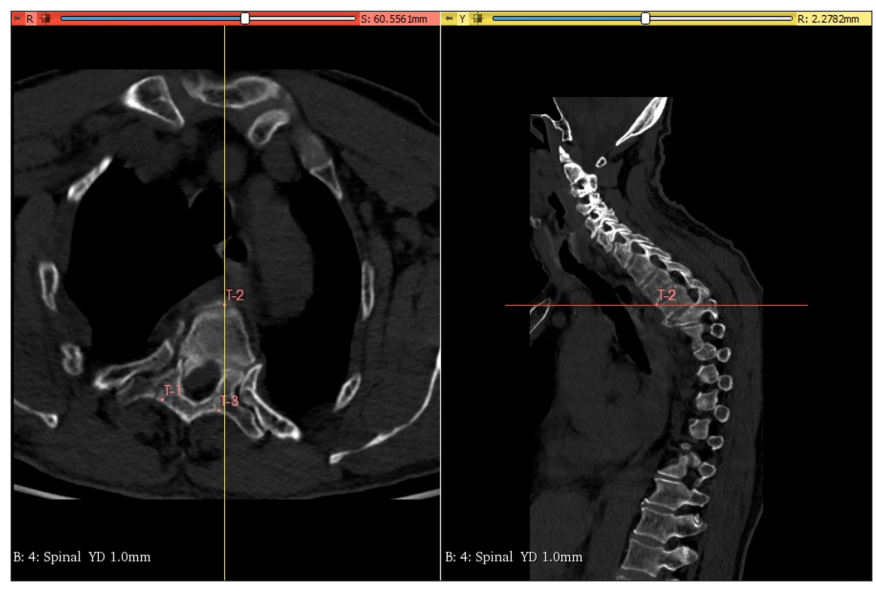

Figure 2. Selecting the desired vertebral level in 3D Slicer

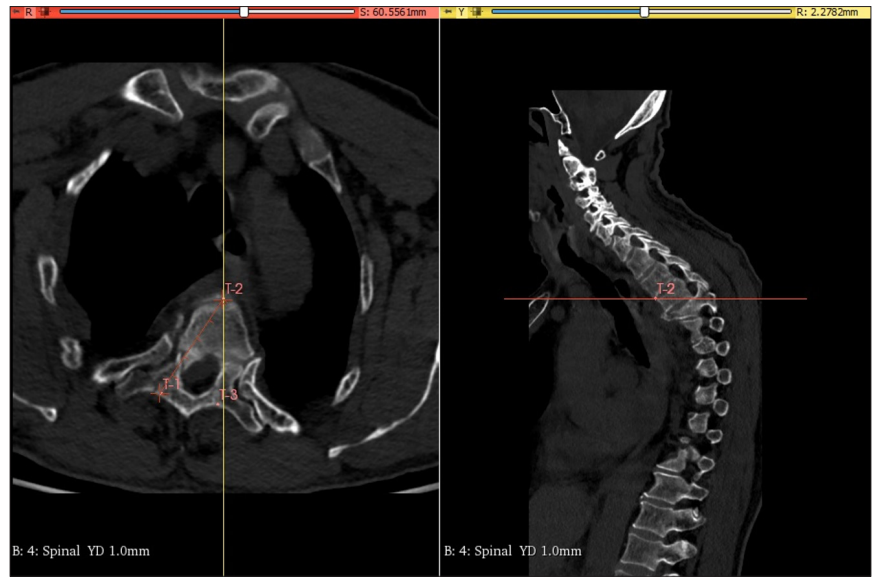

Figure 2. Calculation of Euclidean distance between reference points 
On the other hand, in EA mode, the appropriate entry and reference points were selected on the panels through axial, sagittal, and coronal series and $3 \mathrm{D}$ vertebra models. Then, we entered the desired slope, deviation angle, length, and diameter values of the pedicle screw into the PSS control panel. The "insert the screw for given angle" tab button was activated to visualize the desired pedicle screw (Figure 4).

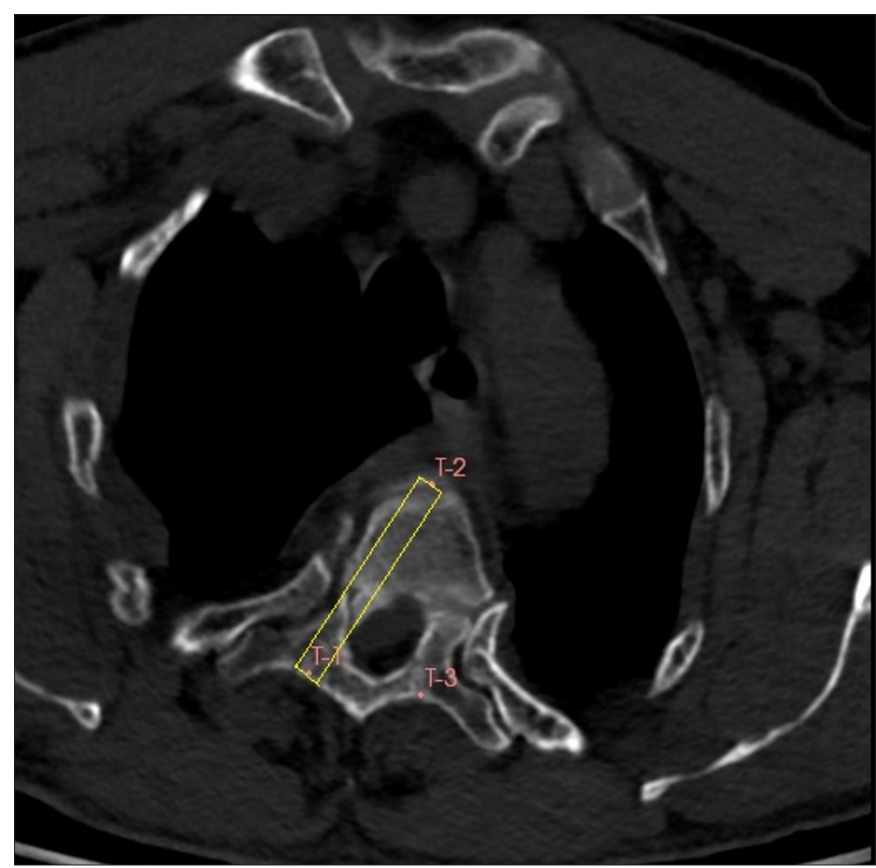

Figure 4. Visualization of the desired pedicle screw

\section{Operational Methods}

We placed pedicle screws through pre-determined anatomic regions. In the other group, pedicle screws were placed under fluoroscopic control.

\section{Evaluation}

The amount of intraoperative bleeding, operation times and screw placement success of the patients were recorded. We then performed control CT scans after the operations (Figure 5, 6). We recorded screw malpositions evaluated them using Rao et al's classification (13). The grading scale is evaluated as follows: $0=$ no perforation of the pedicle; $1=<2 \mathrm{~mm}$ pedicle perforation with one screw thread out of the pedicle; $2=2-4 \mathrm{~mm}$ pedicle perforation; $3=>4 \mathrm{~mm}$ pedicle perforation.

\section{Statistical Analysis}

We analyzed the data using the SPSS (version 20, IBM Inc., Armonk, USA) program. Quantitative data were presented as means and standard deviations, while qualitative data were given as percentages. We performed Chi-square to compare the categorical variables. we considered a $\mathrm{p}$-value of $<0.05$ statistically significant.

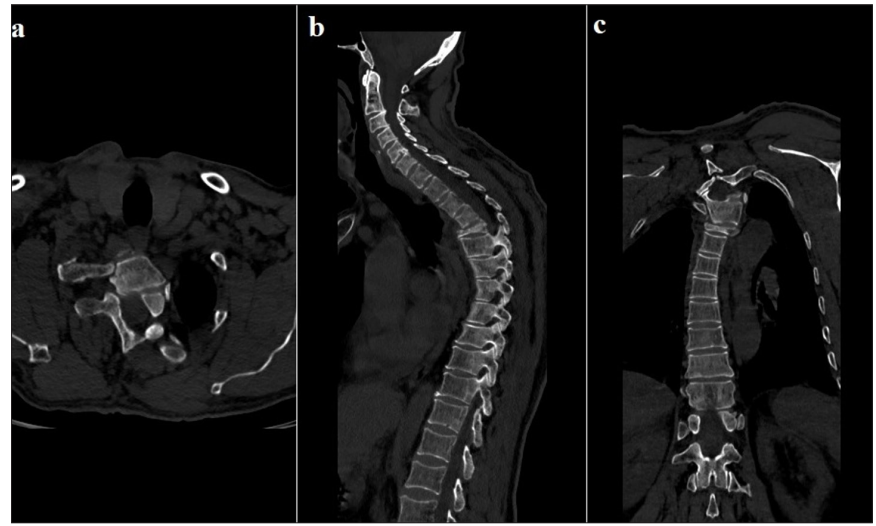

Figure 5. Preoperative CT images

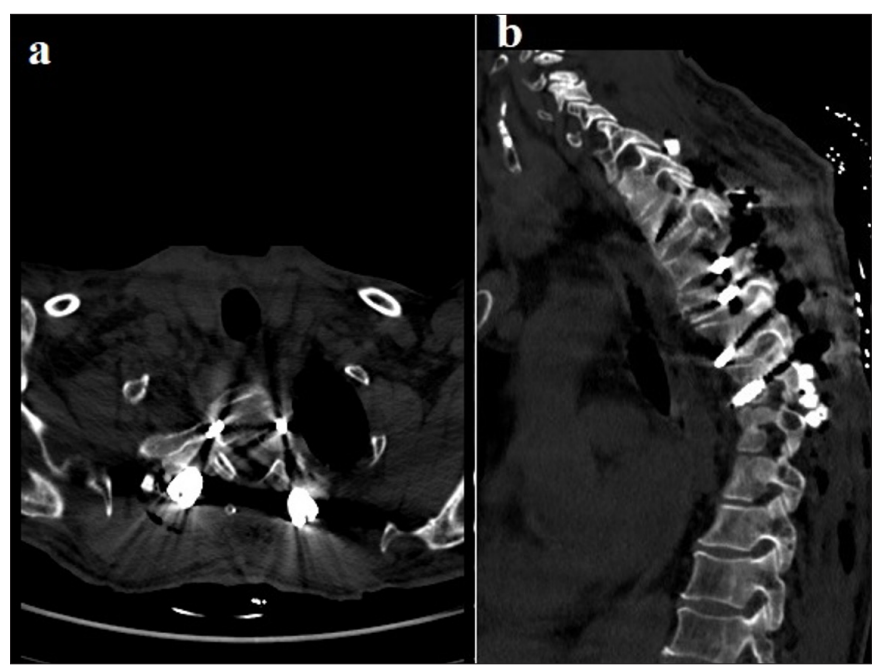

Figure 6. Postoperative CT images

\section{RESULTS}

We studied 24 patients (mean age $=32.3 \pm 4.1$ years, 14 (58.3\%) were male, preoperative PSS was performed in $12(50 \%)$ patients. There were no statistically significant differences between the groups by age and gender $(p>0.05)$ (Table 1). We applied 80 pedicle screws to Group 1 and 72 to Group 2. According to the malposition classification by Rao et al. on postoperative CTs, 68 patients in Group 1 were classified as Grade 0, 8 as Grade 1, 4 as Grade 2. When it comes to the control group (Group 2), 61 patients were classified as Grade 0, 6 as Grade 1, 4 as Grade 2, and 1 as Grade 3. Yet, there were no statistical differences between the groups by Rao classification ( $\mathrm{p}>0.05)$ (Table 2).

\begin{tabular}{|c|c|c|c|}
\hline & Group 1 & Group 2 & p-value $t$ \\
\hline $\begin{array}{l}\text { Number of } \\
\text { patients }\end{array}$ & 12 & 12 & - \\
\hline Sex & 8 males $/ 4$ females & 6 males $/ 6$ females & 0.234 \\
\hline Age & $35.3 \pm 4.3$ & $29.3 \pm 3.9$ & 0.196 \\
\hline
\end{tabular}


Table 2. Rao et al's malposition classification on postoperative CTs

\begin{tabular}{|lcc|}
\hline & $\begin{array}{c}\text { Group 1 } \\
(\mathbf{n = 8 0} \\
\text { screws) }\end{array}$ & $\begin{array}{c}\text { Group 2 } \\
(\mathbf{n}=72 \\
\text { screws) }\end{array}$ \\
\hline Grade 0 (completely within the pedicle) & 68 & 61 \\
Grade 1 (perforation $<2 \mathrm{~mm}$ ) & 8 & 6 \\
Grade 2 (perforation between 2-4 mm) & 4 & 4 \\
Grade 3 (perforation $>4 \mathrm{~mm})$ & - & 1 \\
Accuracy & $95 \%$ & $93.1 \%$ \\
\hline T Accuracy $=$ (Grade 0 + Grade 1)/n*\%100 & & \\
\hline
\end{tabular}

While the mean operation time of Group 1 was $138 \pm 34$ minutes, it was $162 \pm 44$ minutes in Group 2. The groups significantly differed by operation time $(\mathrm{p}<0.05)$ (Table 3). Mean blood loss amounts were $662 \pm 92 \mathrm{~mL}$ in Group 1 and $732 \pm 88 \mathrm{~mL}$ in Group 2 ( $>0.05$ ) (Table 3).

\begin{tabular}{|c|c|c|c|}
\hline & $\begin{array}{c}\text { Group } 1 \\
(n=12)\end{array}$ & $\begin{array}{c}\text { Group } 2 \\
(n=12)\end{array}$ & p value $\boldsymbol{T}$ \\
\hline Operation time (min) & $138 \pm 34$ & $162 \pm 44$ & $\mathrm{p}<0.05$ \\
\hline Blood loss (mL) & $662 \pm 92$ & $732 \pm 88$ & $\mathrm{p}>0.05$ \\
\hline
\end{tabular}

\section{DISCUSSION}

The developments in spine surgery have contributed to the common use of the posterior thoracic interpedicular screwing method today. Due to the complexity of the anatomy of the thoracic pedicles, screw placement is difficult, especially in thoracic fractures. Thoracic pedicles are thin, short, narrow and fragile, causing easy fracture of thoracic pedicles during screwing (14).

Malposition rates can reach $30-40 \%$ in thoracic pedicle screw placement (15). In general, pedicle screw placement is assisted with intraoperative aids such as $\mathrm{C}$-arm fluoroscopy and computed tomography-guided (CT) navigation (6). Besides, CT-guided navigation is costly, so not widely adopted in our country (16). Instead, pedicle screws are commonly placed with the help of C-arm fluoroscopy. However, intraoperative fluoroscopy in long-level fusion surgeries leads surgeons and patients to be exposed to radiation for a longer time (17), which creates a significant problem.

3D Slicer is free and visualization software. This program has an extension called pedicle screw simulator (PSS), which is used in spinal surgery planning. The software allowed us to determine the entry point, direction, diameter, and length of pedicle screws under the guidance of the Fennell technique before spinal surgery; we effectively simulated the Fennell technique on PSS.

In their study, Swaminathan et al. (18) simulated the placement of 120 thoracic pedicle screws on the preoperative PSS. They reported that the simulation allowed successful screw placement both in the craniocaudal and medial-lateral directions. In our study, we concluded that PSS was useful for surgeons, especially for understanding the craniocaudal angle when placing pedicle screws.

In the literature, there were high malposition rates in upper thoracic interpedicular screwing applied through C-arm fluoroscopy $(19,20)$. Karagöz et al. (19) a study of 24 patients retrospectively analyzed 113 thoracic pedicle screws. As a result, they found the rate of incorrect pedicle screw placement to be $20.3 \%$. Also, Vaccaro et al. found the rate of incorrect screw placement to be $41 \%$ on postoperative CT scans (20). In our study, the malposition rates in both the patient group (15\%) and the control group (15.3\%) were lower than those in the literature. In our study, there was no significant difference between the groups in terms of malposition rate ( $p>0.05)$.

The relevant literature proposes that the use of preoperative simulation reduces the surgeon's margin of error and operation time (16). In our study, we found that operation time was significantly shorter in the patient group $(\mathrm{p}<0.05)$. Although the amount of bleeding was less in the patient group, the difference was not statistically significant ( $p>0.05$ ). Further largescale research may obtain significant results on bleeding amount and surgery duration.

It is known that complications occur in inserting pedicle screws, especially in the early learning period (21). In this context, the greatest advantage of such simulators may be that they allow novice surgeons and medical students to make effective pre-operative plans.

This study inevitably bears limitation. The sample size remained relatively small in this study. Hence, largescale studies may be needed to conclude more robust results.

\section{CONCLUSION}

Overall, we concluded that pre-operative simulation on PSS may allow more efficient and easier thoracic pedicle screw application. In addition, the simulator may contribute to the training of surgeons on upper thoracic pedicle screw application and increase the accuracy of pedicular screw placement. The present study may guide further studies with diverse samples and simulators with more advanced technology.

\section{ETHICAL DECLARATIONS}

Ethics Committee Approval: The study was carried out with the permission of Usak University NonInterventional Clinical Researchs Ethics Committee (Date: 23.09.2021, Decision No: 175-175-09).

Informed Consent: All patients signed the free and informed consent form. 
Referee Evaluation Process: Externally peer-reviewed. Conflict of Interest Statement: The authors have no conflicts of interest to declare.

Financial Disclosure: The authors declared that this study has received no financial support.

Author Contributions: All of the authors declare that they have all participated in the design, execution, and analysis of the paper, and that they have approved the final version.

Acknowledgment: The author is very thankful to all the associated personnel in any reference that contributed to the research.

\section{REFERENCES}

1. Cho W, Cho SK, Wu C. The biomechanics of pedicle screw-based instrumentation. J Bone Joint Surg Br 2010; 92: 1061-5.

2. Dede O, Ward WT, Bosch P, Bowles AJ, Roach JW. Using the freehand pedicle screw placement technique in adolescent idiopathic scoliosis surgery: what is the incidence of neurological symptoms secondary to misplaced screws? Spine (Phila Pa 1976) 2014; 39: 286-90.

3. Park SM, Shen F, Kim HJ, et al. How many screws are necessary to be considered an experienced surgeon for freehand placement of thoracolumbar pedicle screws?: analysis using the cumulative summation test for learning curve. World neurosurgery 2018; 118: e550-e6.

4. Perna F, Borghi R, Pilla F, Stefanini N, Mazzotti A, Chehrassan M. Pedicle screw insertion techniques: an update and review of the literature. Musculoskeletal Surg 2016; 100: 165-9.

5. Esses SI, Sachs BL, Dreyzin V. Complications associated with the technique of pedicle screw fixation. A selected survey of ABS members. Spine (Phila Pa 1976) 1993; 18: 2231-8.

6. Cui G, Wang Y, Kao TH, et al. Application of intraoperative computed tomography with or without navigation system in surgical correction of spinal deformity: a preliminary result of 59 consecutive human cases. Spine (Phila Pa 1976) 2012; 37: 891900

7. Xiang L, Zhou Y, Wang H, et al. Significance of preoperative planning simulator for junior surgeons' training of pedicle screw insertion. J Spinal Disord Tech 2015; 28: E25-9.

8. Song G BH, Zhao Y, Han J, Liu Preoperative planning and simulation for pedicle screw insertion using computed tomography-based patient specific volume rendering combined with projection fluoroscopy. Int Robot Autom J 2017; 2: 25-9.

9. Park SK, Yeom JS, Won JH, et al. C1-2 fixation using polyaxial screws and rods assisted by computer simulation for revision of failed posterior fusion: a technical report. jkoa 2017; 40: 778-81.

10. Fedorov A, Beichel R, Kalpathy-Cramer J, et al. 3D slicer as an image computing platform for the quantitative imaging network. Magn Reson Imaging 2012; 30: 1323-41.

11. Muralidharan V, Swaminathan G, Devadhas D, Joseph BV. Patientspecific interactive software module for virtual preoperative planning and visualization of pedicle screw entry point and trajectories in spine surgery. Neurol India 2018; 66: 1766-70.

12. Fennell VS, Palejwala S, Skoch J, Stidd DA, Baaj AA. Freehand thoracic pedicle screw technique using a uniform entry point and sagittal trajectory for all levels: preliminary clinical experience. J Neurosurg Spine 2014; 21: 778-84.

13. Rao G, Brodke DS, Rondina M, Dailey AT. Comparison of computerized tomography and direct visualization in thoracic pedicle screw placement. J Neurosurg 2002; 97: 223-6.
14. Mattei TA, Meneses MS, Milano JB, Ramina R. "Free-hand" technique for thoracolumbar pedicle screw instrumentation: critical appraisal of current "state-of-art". Neurol India 2009; 57: 715-21.

15. Kim YJ, Lenke LG, Bridwell KH, Cho YS, Riew KD. Free hand pedicle screw placement in the thoracic spine: is it safe? Spine (Phila Pa 1976) 2004; 29: 333-42; discussion 42.

16. Shin BJ, James AR, Njoku IU, Hartl R. Pedicle screw navigation: a systematic review and meta-analysis of perforation risk for computer-navigated versus freehand insertion. J Neurosurg Spine 2012; 17: 113-22.

17. Rampersaud YR, Foley KT, Shen AC, Williams S, Solomito M. Radiation exposure to the spine surgeon during fluoroscopically assisted pedicle screw insertion. Spine (Phila Pa 1976) 2000; 25: 2637-45.

18. Swaminathan G, Muralidharan V, Devakumar D, Joseph BV. Accuracy of the freehand (fennell) technique using a uniform entry point and sagittal trajectory for insertion of thoracic pedicle screws: A computed tomography-based virtual simulation study. Neurol India 2020; 68: 468-71.

19.Guzey FK, Emel E, Hakan Seyithanoglu M, et al. Accuracy of pedicle screw placement for upper and middle thoracic pathologies without coronal plane spinal deformity using conventional methods. J Spinal Disord Tech 2006; 19: 436-41.

20. Vaccaro AR, Rizzolo SJ, Balderston RA, et al. Placement of pedicle screws in the thoracic spine. Part II: An anatomical and radiographic assessment. J Bone Joint Surg Am 1995; 77: 1200-6.

21.Wi W, Park SM, Shin BS. Computed tomography-based preoperative simulation system for pedicle screw fixation in spinal surgery. J Korean Med Sci 2020; 35: e125. 